\title{
Preliminary results of variable star search using KMTNet data
}

\author{
Young-Beom Jeon * \\ ${ }^{1}$ Korea Astronomy and Space Science Institute 776, Daedeokdae-ro, Yuseong-gu, Daejeon, 34055, \\ Republic of Korea
}

\begin{abstract}
The KMTNet (Korea Microlensing Telescope Network) was completed on October 2nd in 2015. There are three $1.6 \mathrm{~m}$ telescopes in Chile (CTIO), Australia (SSO) and South Africa (SAAO). The field of view of the CCD camera is 2 degree $\times 2$ degree. We are monitoring and observing the bulge region for 24-hour continuous observations using the KMTNet telescopes. I present the characteristics of CCD camera and telescope, and the status of observation programs. We are still developing CCD cameras to accurate photometry. From the KMTNet data, we can obtain accurate 24-hour continuous time-series data for many variable stars.
\end{abstract}

\section{Introduction}

The Korea Microlensing Telescope Network (KMTNet) is a wide-field photometric system composed by three identical $1.6 \mathrm{~m}$ telescopes. The most important capability of the KMTNet is 24 hours continuous observations using the three telescopes installed at three different continents; CTIO in Chile, South America, SSO in Australia and SAAO in South Africa. The primary science of the KMTNet is the Galactic bulge monitoring to find extrasolar planets. The monitoring data are very useful to find microlensing events, variable stars, and supernovae, etc. The science programs and the system specifications of the KMTNet are well explained in [2]. Table 1 presents brief specifications for telescopes and CCD camera. The first results for the microlensing effects and a variable star monitoring are published in [3] and [1], respectively.

\section{Preliminary results of variable star search}

Figure 1 shows sample images and preliminary results of variable star search. There are 1,600 small regions in a $\mathrm{N}$ chip. I checked 20 regions at first. From all 20 regions, I found above 100 variable stars including short period $\delta$ Sct, eclipsing binary, RR Lyrae, Cepheid and long periodic regular or semi-regular variable stars. We expect to detect planet transits, too. Most images are obtained in the $I$-band, and also $V$-band to determine the color, occasionally.

Till now, we are developing image processing procedures and photometry methods. We will use the pieces of 1024 pixels $\times 1024$ pixels for image subtraction processes after 2017 .

Acknowledgments: This work was supported by KASI (Korea Astronomy and Space Science Institute) grant 2016-1-832-01.

^ybjeon@kasi.re.kr 


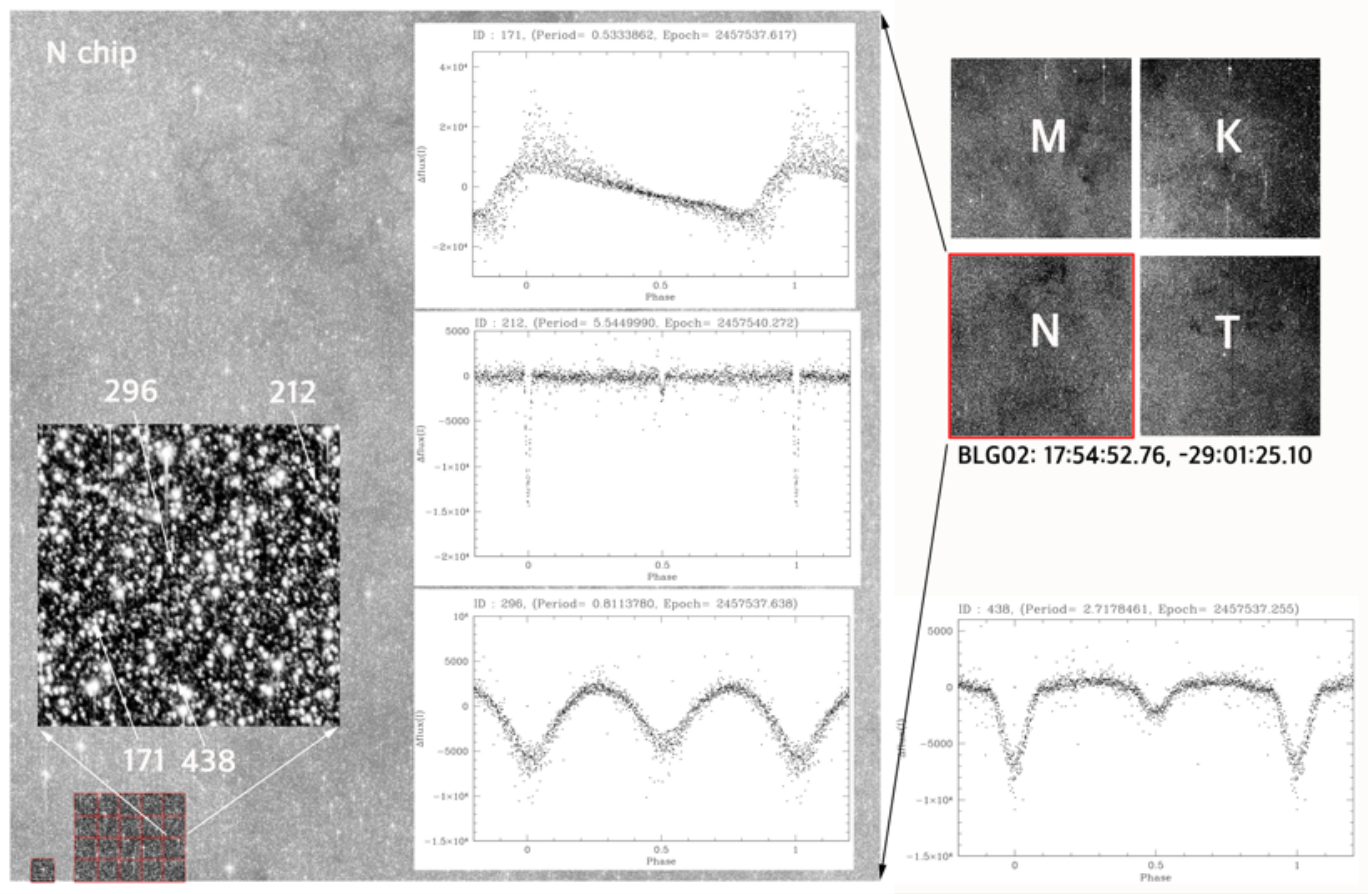

Figure 1. Preliminary results of variable star search using the KMTNet data. The whole K, M, T, N mosaic field is shown on the upper right side. To find variable stars, I selected a few small regions of the $\mathrm{N}$ chip field. Four variable stars were found from a small sample region which has 256 pixels $\times 256$ pixels.

Table 1. Specifications of the KMTNet system.

\begin{tabular}{lll}
\hline Telescope & Location & CTIO Chile, SSO Australia, SAAO South Africa \\
& Diameter & $1.6 \mathrm{~m}$, each \\
& FOV & 2 degree $\times 2$ degree \\
& Focal length & $5160 \mathrm{~mm}$ \\
& Focus & Prime \\
CCD camera & & \\
& Manufacture & $\mathrm{e} 2 \mathrm{v}$ \\
& Mosaic & 4 ea $\times 9 \mathrm{k} \times 9 \mathrm{k}$ \\
& Plate scale & $10 \mu \mathrm{m} /$ pixel $\times 10 \mu \mathrm{m} /$ pixel, $0.4^{\prime \prime} /$ pixel \\
& Filters & Johnson-Cousins $B V R I$, SDSS griz, $H_{\alpha}$ \\
\hline
\end{tabular}

\section{References}

[1] Kim, S.-L., Cha, S.-M., Lim, B., et al., JKAS, 49, 199 (2016)

[2] Kim, S.-L, Lee, C.-U., Park, B.-G., et al., JKAS, 49, 37 (2016)

[3] Shin, I.-G. Ryu, Y.-H., Udalski, A., et al., JKAS, 49, 73 (2016) 